\title{
H.264/MPEG-4 AVC Video Streaming Evaluation of LR-EE-AOMDV Protocol in MANET
}

\author{
Periyasamy Pitchaipillai ${ }^{1}$ and Karthikeyan Eswaramoorthy ${ }^{2}$ \\ ${ }^{1}$ Department of Computer Science and Applications, Sree Saraswathi Thyagaraja College, Pollachi, Tamil Nadu, India \\ ${ }^{2}$ Department of Computer Science, Government Arts College, Udumalpet, Tamil Nadu, India
}

\begin{abstract}
Deployment of conversational real-time applications like VoIP (Voice over IP) and videoconferencing require new challenges in Long-Term Evolution (LTE) networks such as Internet. Quality of video delivered over the Internet has been determined in terms of Quality-of-Service (QoS) and Quality-of-Experience (QoE). QoS includes packet delivery ratio, average end-to-end delay, throughput, routing overhead, etc. QoE is a qualitative measure of videos transmitted over Internet. In this paper, we evaluate the performance of Link Reliable Energy Efficient Ad hoc On-demand Multipath Distance Vector (LR-EE-AOMDV) routing on H.264/MPEG-4 AVC video streaming under both Distributed Coordination Function (DCF) and Enhanced Distributed Coordination Function (EDCF) using NS2.34 and Enhanced EvalVid framework. Simulation results show that the LR-EE-AOMDV performs better than AOMDV and OMMRE-AOMDV.
\end{abstract}

ACM CCS (2012) Classification: Networks $\rightarrow$ Network protocols $\rightarrow$ Network layer protocols $\rightarrow$ Routing protocols

Networks $\rightarrow$ Network performance evaluation $\rightarrow$ Network simulation

Networks $\rightarrow$ Network types $\rightarrow$ Ad hoc networks $\rightarrow$ Mobile ad hoc networks

Keywords: MANET, multipath routing, QoS, QoE, VoIP, AOMDV, LR-EE-AOMDV, H.264/MPEG-4 AVC, LTE, DCF, EDCF

\section{Introduction}

In this modern world, wireless communication has become indispensable part of life. Research focuses on Mobile Ad hoc NETworks (MANETs), which is a collection of mobile devices by wireless links forming a dynamic topology without much physical network infrastructure such as routers, servers, access points/ cables or centralized administration. Each mobile device functions as a router as well as a node. The main characteristics of MANET are i) dynamic topologies ii) bandwidth-constrained links iii) energy constrained operation and iv) limited physical security [1], [2].

Long-Term Evolution (LTE) is a standard developed by the Third Generation Partnership Project (3GPP) for high-speed data service in wireless communication by providing increased peak data rates of $100 \mathrm{Mbps}$ downstream and 50 Mbps upstream [3], [4]. Nowadays, telecommunication systems are supporting different kinds of real-time transmission; video transmission is one of the most important applications. The quality of the supported video in such network is a major issue due to the increasing deployment of conversational real-time applications like VoIP (Voice over IP) and videoconferencing. The quality of experience (QoE) is defined by ITU-T [5] as "the overall acceptability of an application or service, as perceived by the end-user"; this does not only include QoS, but also considers the capability of user equipment (UE) as well as the user's expectation and context. H.264/MPEG-4 Advanced Video Coding (AVC) [6] is the latest widely accepted international video coding standard. It was jointly developed by the Video Coding Experts Group (VCEG) of the International Telecommunication Union-Telecommunication (ITU-T) and the Moving Picture Experts Group (MPEG) of the International Organization for Standardiza- 
tion (ISO)/International Electrotechnical Commission (IEC). Today's application areas are ranging from videoconferencing over mobile TV and broadcasting of standard-/high-definition TV content up to very-high-quality applications such as professional digital video recording or digital cinema/large-screen digital imagery using H.264/MPEG-4 AVC standards for transmission of videos due to its so-called fidelity range extensions (FRExt) which enables new services and thus opens new markets and opportunities for the industry. In this paper, we investigate the performance of Link Reliable Energy Efficient Ad hoc On-demand Multipath Distance Vector (LR-EE-AOMDV) routing on H.264 video streaming under Distributed Coordination Function (DCF) or Distributed Channel Access (DCA) and Enhanced Distributed Coordination Function (EDCF) or Enhanced Distributed Channel Access (EDCA) using NS2.34 and enhanced EvalVid framework.

The rest of the paper is organized as follows: Section 2 presents the overview of Link Reliable Energy Efficient Ad hoc On-demand Multipath Distance Vector (LR-EE-AOMDV) routing. In Section 3, we present the overview of EvalVid framework. In Section 4, the Video Quality Metrics such as Quality-of-Service (QoS) and Quality-of-Experience (QoE) are explained. In Section 5, the simulation and experimental results are discussed. In Section 6, the conclusions and future work are presented.

\section{Overview of AOMDV, OMMRE-AOMDV and LR-EE-AOMDV Routing}

Ad hoc on-demand multipath distance vector (AOMDV) [7] is a multipath extension of a prominent and well-studied on-demand single path routing protocol known as ad hoc on-demand distance vector (AODV) [8]. AOMDV eliminates the occurrence of frequent link failures and route breaks in highly dynamic ad hoc networks due to node mobility, node failures, congestion in traffic, packet collisions, and so on, by adding some extra fields in routing tables and control packets in order to compute loopfree and link-disjoint multiple routes between the source and destination.
Routing Process of AOMDV has three phases such as

(i) route discovery,

(ii) route selection, and

(iii) route maintenance.

In AOMDV, the RREQ (Route REQuest), RREP (Route REPly) or HELLO packets are transmitted over links of nodes in order to establish, select and maintain routes between any source and destination. These packets are called Received Signal Strength Indicators (RSSI) [9]. In AOMDV, the propagation of RREQs from a source to a destination via intermediate nodes is used to establish multiple reverse routes. The propagation of RREPs from a destination to a source via intermediate nodes is used to establish multiple forward routes and the flooding of HELLO packets between nodes used mainly to obtain local link connectivity after route establishment [10]. Every node locally updates its routing table upon receiving HELLO packets, called Local Path Update (LPU).

AOMDV provides link-disjoint, loop free and fault tolerance paths which improve the network lifetime by minimizing packet loss, routing overhead and energy consumption. However, it does not ensure link reliability due to the selection of multiple paths based on only hop count. The AOMDV routing protocol updates its routing table periodically on-demand upon receiving RREQ/RREP based on the route update rules shown in Algorithm 1 [7]. Whenever a node $i$ receives a route advertisement to a destination $d$ from a neighbor $j$, it invokes AOMDV path discovery process in order to set up forward as well as reverse routes, as shown in Algorithm 1.

In Algorithm 1 the variables seqnum ${ }_{i}{ }^{d}$, advertised_hopcount ${ }_{i}^{d}$ and route_list ${ }_{i}^{d}$ represent the sequence number, advertised hop count and route list for destination $d$ at node $i$ or node $j$ respectively.

Figure 1 illustrates the route selection process of AOMDV protocol. Here, S and D are the source and the destination, respectively. The path $\mathrm{S}-\mathrm{C}-\mathrm{G}-\mathrm{D}$ with Hops $=2$ is selected as a primary route for data transmission, the paths $\mathrm{S}-\mathrm{A}-\mathrm{F}-\mathrm{H}-\mathrm{D}$ with Hops $=3$ and $\mathrm{S}-\mathrm{B}-$ $\mathrm{E}-\mathrm{I}-\mathrm{D}$ with Hops $=3$ are chosen as alternate routes. The main disadvantage of AOMDV is 
Algorithm 1. Route update rules of AOMDV protocol [7].
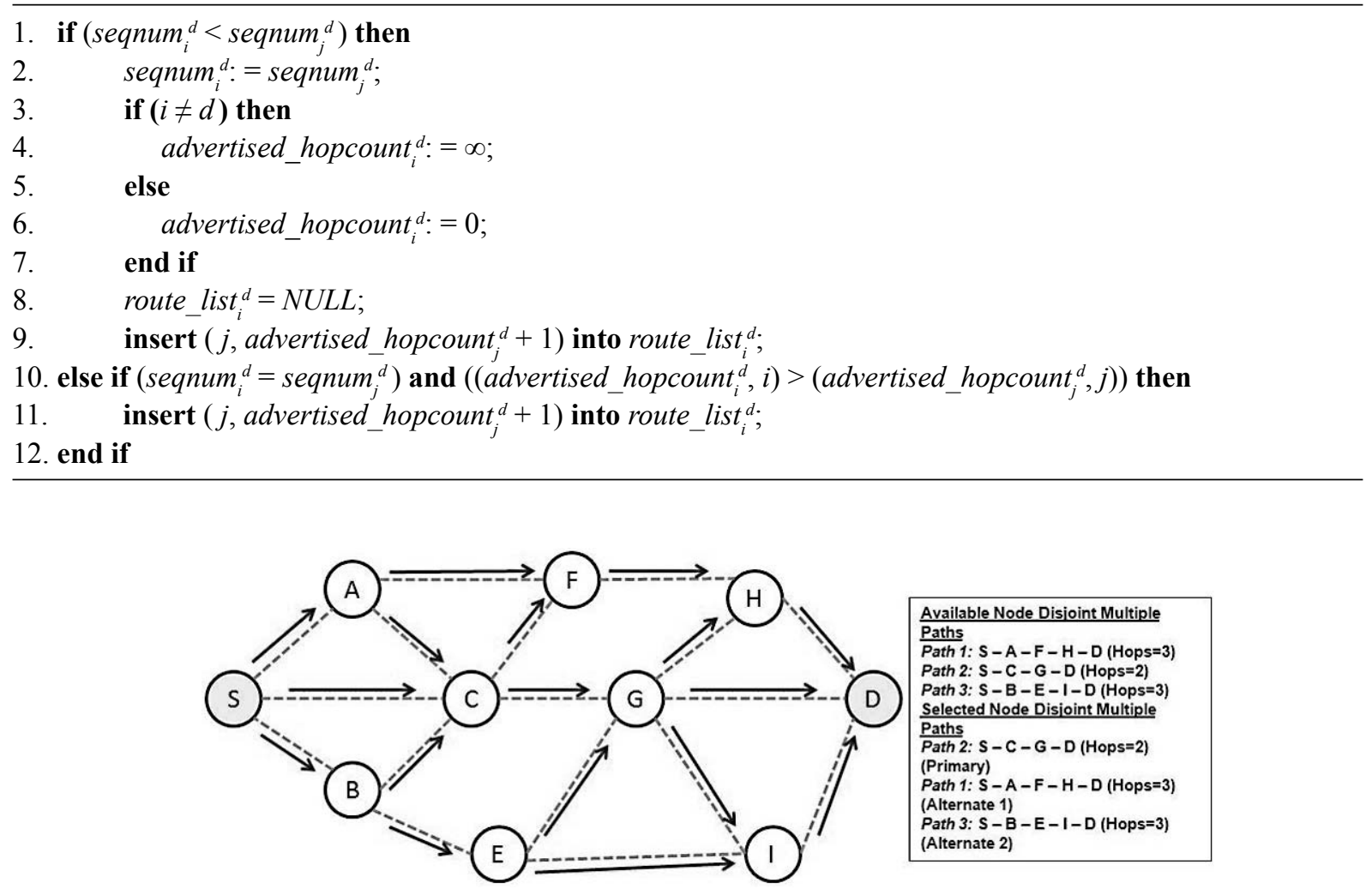

Figure 1. Route Selection Process of AOMDV protocol [7].

that the routes are selected only based on minimal hop count (traditional route selection metrics) which does not ensure reliable data transmission since it does not eliminate the link and node failures.

Optimized minimal maximal residual energy AOMDV (OMMRE-AOMDV) [11] is an enhanced version of minimal maximal residual energy AOMDV (MMRE-AOMDV) [12] which improves network lifetime by provid- ing more energy-efficient, link-disjoint, loop free and fault tolerance paths by finding minimal residual energy of each path without taking the destination into account, better than MMRE-AOMDV and AOMDV. The route update rules of OMMRE-AOMDV are shown in Algorithm 2 and the structure of routing table entries of AOMDV, MMRE-AOMDV and OMMRE-AOMDV routing protocols are shown in Table 1. When a node $i$ receives a RREQ or RREP, it updates its advertised hop count for

Table 1. Structure of routing table entries of AOMDV, MMRE-AOMDV and

OMMRE-AOMDV routing protocols.

\begin{tabular}{|c|}
\hline destination \\
\hline sequence number \\
\hline advertised hop count \\
\hline $\begin{array}{c}\text { route list } \\
\left(\text { nexthop }_{1}, \text { hopcount }_{1}\right), \\
\left.\left(\text { nexthop }_{2}, \text { hopcount }_{2}\right), \ldots\right\}\end{array}$ \\
\hline expiration time out \\
\hline
\end{tabular}

AOMDV [7]

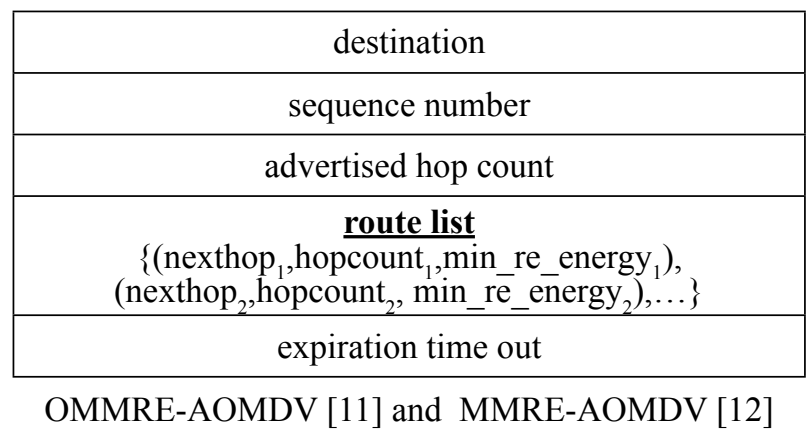


Algorithm 2. Route update rules of OMMRE-AOMDV routing protocol [11].

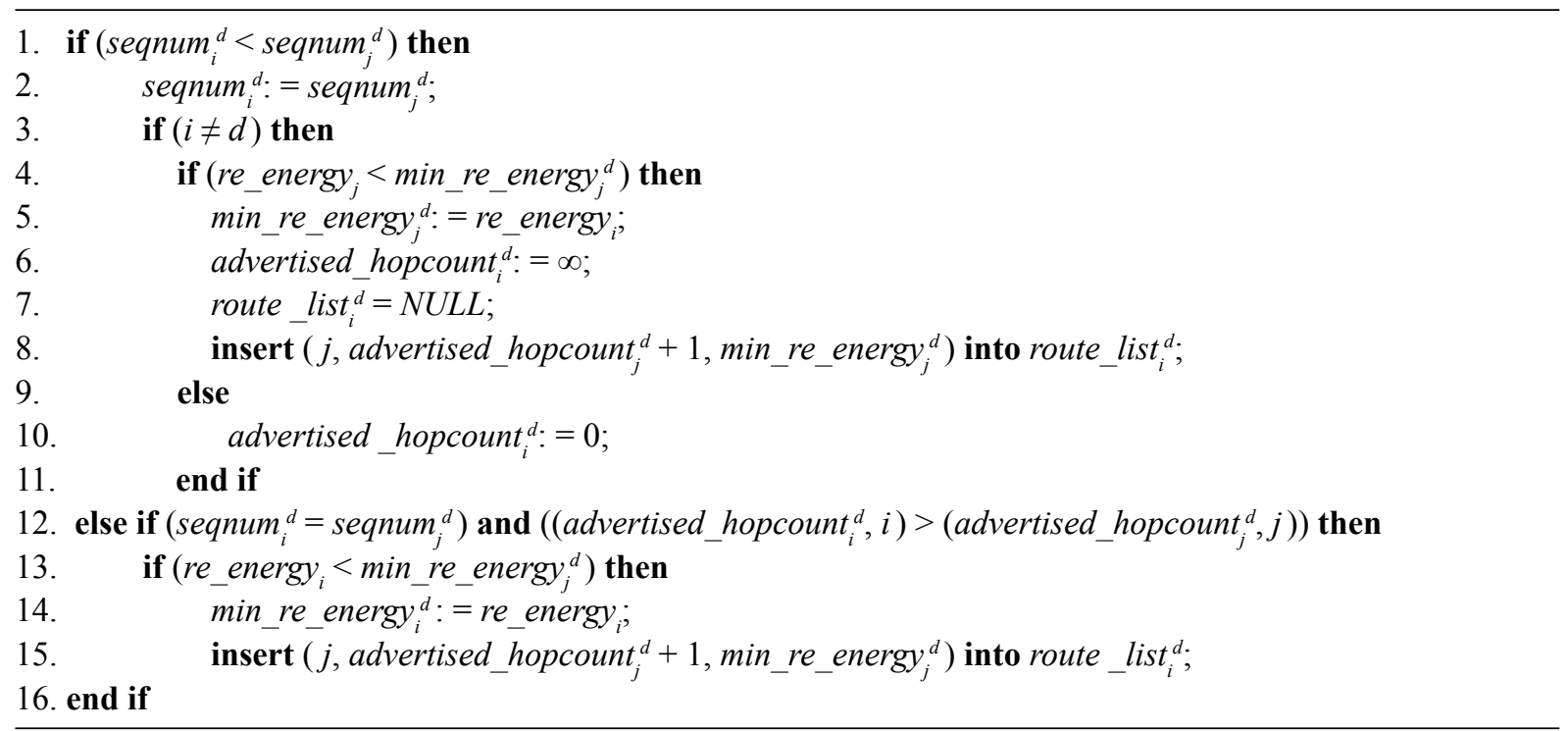

a destination $d$ if its sequence number is less than the sequence number of RREQ or RREP of node $j$. It also updates its minimal residual energy with the minimal residual energy of RREQ or RREP of node $j$ if minimal residual energy of RREQ or RREP of node $i$ is less than minimal residual energy of RREQ or RREP of node $j$.

Whenever a node $i$ receives a route advertisement to a destination $d$ from a neighbor $j$, it invokes OMMRE-AOMDV path discovery process in order to set up forward as well as reverse routes as shown in Algorithm 2. In Algorithm 2 the variables seqnum ${ }_{i}^{d}$, advertised_hopcount ${ }_{i}^{d}$, route_listid, re_energy ${ }_{i}^{d}$ and min_re_energy ${ }_{i}^{d}$ represent the sequence number, advertised hop count, route list, residual energy and minimal residual energy for the destination $d$ at node $i$ or node $j$ respectively.
Figure 2 illustrates the route selection process of OMMRE-AOMDV protocol. Here, the number in each rectangle is the residual energy of that node and S and D are the source and the destination, respectively. The path $\mathrm{S}-\mathrm{C}-\mathrm{G}-$ $\mathrm{D}$ with Hops $=2$ and the highest minimal residual energy $(\mathrm{MRE})=70$ are selected as a primary route for data transmission, the path $\mathrm{S}-\mathrm{A}-\mathrm{F}$ $-\mathrm{H}-\mathrm{D}$ with Hops $=3$ and the next highest $\mathrm{MRE}=50$ are chosen as alternate route and the path $\mathrm{S}-\mathrm{B}-\mathrm{E}-\mathrm{I}-\mathrm{D}$ with Hops $=3$ and are next highest $\mathrm{MRE}=20$ the chosen as next alternate route. The main disadvantage of OMMRE-AOMDV is that the routes are selected only based on minimal hop count (traditional route selection metrics) and maximized minimal residual energy, which does not ensure reliable data transmission since it does not elim-

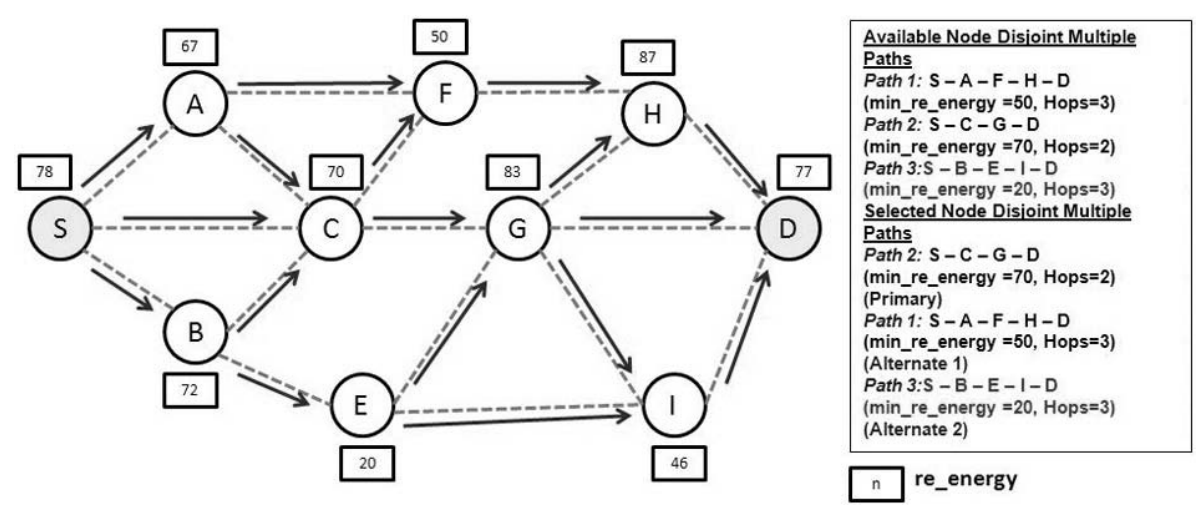

Figure 2. Route selection process of OMMRE-AOMDV protocol [11]. 
inate the link failures, but it eliminates node failures by generating energy efficient routes between the source and the destination.

Quality of wireless links is determined by estimating the number of transmissions and retransmissions needed to send a data packet over a link between nodes, called Expected Transmission Count (ETX) [13] - [14] of that link. The summation of the ETX of all participating links of the route is called path ETX or PathLink Quality Estimator (P-LQE) or Cumulative Expected Transmission Count (CETX). Received Signal Strength Indicator (RSSI) is determined initially by RREQ or RREP during route discovery and then by HELLO packets during route selection and maintenance. Since the RREQ or RREP packets are used to determine the stability of links between nodes during route discovery, they are used to calculate both ETX and CETX in this protocol. In this paper, the ETX of a link between the nodes along the forward path is computed using RREP packets. The ETX of a link between the nodes along the reverse path is computed using RREQ packets.

Quality of wireless nodes is determined by estimating the amount of energy needed by a node to send a data packet to another node over a link, called Expected Transmission Energy (ETE) [15] of that node. The summation of the ETE value of all participating nodes of the route is called path ETE or Path-Node Energy Estimator (P-NEE) or Cumulative Expected Transmission Energy (CETE). The minimal value of the residual energy of the participating nodes of a route is called Minimal Residual Energy (MRE) [12]; this value is used as threshold for CETE during route selection.

Many researchers consider minimal nodal residual energy and hop count as metrics for route selection. When the minimal nodal residual energy of the path does not meet the energy required for data transmission, a node failure occurs during data transmission. All routing protocols use link expiration time (LET) for measuring link stability. When a link is alive (LET of that link is not expired), but is not within the transmission range, it fails to transmit the data. The scope of LR-EE-AOMDV [16] protocol is to measure dynamically Cumulative expected transmission count (CETX) for ensuring link reliability in addition to LET, $\mathrm{Cu}-$ mulative expected transmission energy (CETE) for ensuring minimal transmission energy in addition to minimal nodal residual energy, and path length for finding the shortest paths for data transmission. LR-EE-AOMDV uses three metrics integrated approach to select the link reliable energy efficient shortest path for data transmission. The AOMDV uses the traditional routing metric hop count for finding multiple routes and selects a route with a few hop counts among them for data transmission. During data transmission, if any link between the nodes of that route fails or energy of any node of that route downs, the data loss occurs in AOMDV. To rectify this problem, the LR-EE-AOMDV proposed by modifying AOMDV routing protocol using hop count, CETX and CETE as path selection metrics and the routes are periodically updated based on the route update rules shown in Algorithm 3. Table 2 shows the structure of routing table entries of LR-EE-AOMDV routing protocol.

Whenever a node $i$ receives a route advertisement to a destination $d$ from a neighbor $j$, it invokes LR-EE-AOMDV path discovery process in order to set up forward as well as reverse routes as shown in Algorithm 3. The variables seqnum $_{i}^{d}$, advertised_hopcount ${ }_{i}^{d}$, route list $_{i}^{d}$, cetx $_{i}^{d}$, cete $_{i}^{d}$, re energy ${ }_{i}^{d}$, initial_energy ${ }_{i}{ }^{d}$,consumed_energy ${ }_{i}{ }^{-}$and mre $_{i}^{d}$ represent the sequence number, advertised hop count, route list, cumulative expected transmission count, cumulative expected transmission energy, residual energy, initial energy, consumed energy and minimal residual energy for the destination $d$ at node $i$ or node $j$ respectively.

Each RREQ and RREP of LR-EE-AOMDV protocol totally carries three additional fields such as the sum of the ETX value of link over which the RREQ or RREP has traversed, called CETX, the sum of the ETE values of node over which the RREQ or RREP has traversed, called CETE, and the residual energy of the node called re energy shown in Table 3. Similar to AOMDV routing protocol, in LR-EE-AOMDV, when a node receives a RREQ packet for the first time, it rebroadcasts the RREQ packet immediately.

When a source $S$ floods RREQ to a destination $\mathrm{D}$ or a destination $\mathrm{D}$ sends back RREP to a source $\mathrm{S}$, the CETX of RREQ/RREP is ini- 
tialized by 0 , the CETE of RREQ/RREP is initialized by 0 and re_energy of RREQ/RREP of a node is initialized by the current energy. The mre field of the routing table entry of our pro- posed protocol is also initialized by the current energy of the source node during route discovery. Upon receiving the RREQs or RREPs, the intermediate nodes find the ETX value in terms

Algorithm 3. Route update rules of LR-EE-AOMDV [16].

1. $\operatorname{cetx}_{j}^{d}:=\operatorname{cetx}_{j}^{d}+\operatorname{etx}_{j}^{d}$

2. cete $_{j}^{d}:=$ cete $_{j}^{d}+$ ete $_{j}^{d}$;

3. re_energy $y_{j}^{d}:=$ initial_energy $y_{j}^{d}$-consumed_energy ${ }_{j}^{d}$

4. if $\left(r e_{\text {energy }}<m r e_{j}^{d}\right)$ then

5. $\quad$ mre $_{j}^{d}=$ re_energy;

6. end if

7. if $\left(\right.$ seqnum $_{i}^{d}<$ seqnum $\left._{j}^{d}\right)$ then

8. seqnum $_{i}^{d}:=$ seqnum $_{j}$;

9. if $(i \neq d)$ then

10. $\quad \operatorname{cetx}_{i}^{d}:=\operatorname{cetx}_{j}^{d}$

11. cete $_{i}^{d}:=$ cete $_{j}^{d}$;

12. advertised_hopcount $t_{i}^{d}:=\infty$;

13. else

14. advertised_hopcount $t_{i}^{d}=0$;

15. end if

16. route_list ${ }_{i}^{d}:=N U L L$;

17. $\quad$ insert $\left(j\right.$, advertised_hopcount ${ }_{j}^{d}+1$, cetx $_{j}^{d}$, cete $_{j}^{d}$, mre $\left._{j}^{d}\right)$ into route_list ${ }_{i}^{d}$;

18. else if $\left(\right.$ seqnum $_{i}^{d}=$ seqnum $\left._{j}^{d}\right)$ and $\left(\left(\right.\right.$ advertised_hopcount $\left.{ }_{i}^{d}, i\right)>\left(\right.$ advertised_hopcount $\left.\left._{i}^{d}, j\right)\right)$ and $\left(\left(\right.\right.$ cetx $\left._{i}^{d}, i\right)$ $>\left(\right.$ cetx $\left._{j}^{d}, j\right)$ and $\left(\left(\right.\right.$ cete $\left._{i}^{d}, i\right)<$ mre $\left._{j}^{d}\right)$ then

19. $\quad$ insert $\left(j\right.$, advertised_hopcount ${ }_{j}^{d}+1$, cetx $_{j}^{d}$, cete $_{j}^{d}$, mre $\left._{j}^{d}\right)$ into route_list ${ }_{i}^{d}$;

$/ *$ Got a new node disjoint alternate path and insert it into routing table*/

20. if (num_paths ${ }_{i}^{d}=\max$ num_paths $)$ and $\left(\left(\left(\left(\right.\right.\right.\right.$ advertised_hopcount $\left.\left.{ }_{j}^{d}, j\right)+1\right)-\min \left(\left(\right.\right.$ advertised_hopcount $\left.\left._{i}^{d}, i\right)\right)$ $\left.{ }_{i}^{<}<=M A \bar{X} P A T H \_L E N G T H\right)$ and $\left(\left(\operatorname{cetx}_{j}^{d^{-}-\min }\left(\right.\right.\right.$ cetx $\left.\left.\left._{i}^{d}, i\right)\right)<=1.0\right)$ and $\left(\left(\right.\right.$ cete $\left._{i}^{d}, \bar{i}\right)<$ mre $\left._{j}^{d}\right)$ then

21. insert $\left(j\right.$, advertised_hopcount ${ }_{j}^{d}+1$, cetx $_{j}^{d}$, cete $_{j}^{d}$, mre $\left._{j}^{d}\right)$ into route_list ${ }_{i}^{d}$;

22. $\operatorname{cet} x_{i}^{d}:=\operatorname{cetx}_{j}^{d}$;

23. cete $_{i}^{d}:=$ cete $_{j}^{d}$;

24. end if

25. end if

Table 2. Structure of routing table entries of AOMDV and LR-EE-AOMDV routing protocols.

\begin{tabular}{|c|}
\hline destination \\
\hline sequence number \\
\hline advertised hop count \\
\hline $\begin{array}{c}\text { route list } \\
\left\{\text { (nexthop }_{1}, \text { hopcount }_{1}\right), \\
\left.\left(\text { nexthop }_{2}, \text { hopcount }_{2}\right), \ldots\right\}\end{array}$ \\
\hline expiration time out \\
\hline
\end{tabular}

AOMDV [7]

\begin{tabular}{|c|}
\hline destination \\
\hline sequence number \\
\hline advertised hop count \\
\hline $\begin{array}{c}\text { route list } \\
\left(\text { (nexthop }_{1}, \text {,opcount }_{1}, \text { cetx }_{1}, \text { cete }_{1}, \text { mre }_{1}\right), \\
\left.\left(\text { nexthop }_{2}, \text { hopcount }_{2}, \text { cetx }_{2}, \text { cete }_{2}, \text { mre }_{2}\right), \ldots\right\}\end{array}$ \\
\hline expiration time out $^{2}$ \\
\hline
\end{tabular}

LR-EE-AOMDV [16]

Table 3. Extended RREQ/RREP message format [16].

\begin{tabular}{|l|l|l|l|l|l|l|l|}
\hline Source Address & Destination Address & Sequence Number & Hop Count & Time Out & CETX & CETE & re_energy \\
\hline
\end{tabular}


of the number of RREQ or RREP packets over the ends of the link and the ETE value in terms of the energy consumed by a participating node of the path; the CETX as well as the CETE values are updated periodically using Algorithm 3 which deals with the following two cases in order to select the paths based on the three metrics - hop count, CETX and CETE:

Case 1: From Lines 7-17 of Algorithm 3, the intermediate node updates its routing table by updating the CETX and CETE values with the CETX and CETE values of RREQ/RREP of this node respectively if the sequence number of just received packet is greater than this node.

Case 2: From Lines $18-25$ of Algorithm 3, the intermediate node updates its routing table by updating CETX and CETE values with the CETX and CETE values of RREQ/RREP of this node respectively if the sequence number of just received packet is equal to this node and the hop count is less than the hop count of RREQ/RREP and the CETX value of RREQ/ RREP is greater than the CETX value of that intermediate node, the CETE value of RREQ/ RREP being less than the MRE of the path.

Figure 3 illustrates the route selection process of LR-EE-AOMDV protocol. Here, the number in each link is the ETX value of that link, the number in each rectangle is the ETE value of that node, the number in each hexagon is the residual energy of that node, and S and D are the source and the destination, respectively. In LR-EE-AOMDV protocol, the path with CETX value $(\mathrm{CETX}<1$ and CETX $>0)$, CETE value (CETE $>$ MRE) and the minimal hop count are selected for data transmission. When the ETX value of a link between two nodes is zero, it is considered as a weak link which is not consid- ered for data transmission. For example, node F sends 3 RREQ packets to node $\mathrm{H}$ per second, then the $P R R_{\text {forward }(F, H)}$ is 3 and the node $\mathrm{H}$ receives 1 RREQ packet from node $\mathrm{F}$ per second, then the $P R R_{\text {backward }(F, H)}$ is 1 and the ETX value of the link between nodes $\mathrm{F}$ and $\mathrm{H}$ is 0.3 . The path $\mathrm{S}-\mathrm{C}-\mathrm{G}-\mathrm{D}$ with CETX $=0.3$, CETE $=16$, Hops $=2$ and $\mathrm{MRE}=70$ is selected as a primary route for data transmission and the path $\mathrm{S}-\mathrm{A}-\mathrm{F}-\mathrm{H}-\mathrm{D}$ with $\mathrm{CETX}=0.6, \mathrm{CETE}=21$, Hops $=3$ and $\mathrm{MRE}=50$ is chosen as alternate route.

\section{Overview of Enhanced EvalVid Framework}

\subsection{MPEG-4 Video Coding Terminology}

MPEG-4 [17] video sequence comprises of three types of frames such as

(i) I-frames (Intra-coded) - frames in a video clip that are compressed using the combination of various lossless and lossy compression techniques without making reference to any previous or subsequent frame in the sequence. These frames are the largest of the three with highest-quality, but they are the least efficient from a compression perspective. These are also known as key frames.

(ii) P-frames (predicted) - frames in a video clip that are produced by the encoder using backward reference to previous I or Pframes for redundant picture information. P-frames are more efficient than I-frames,

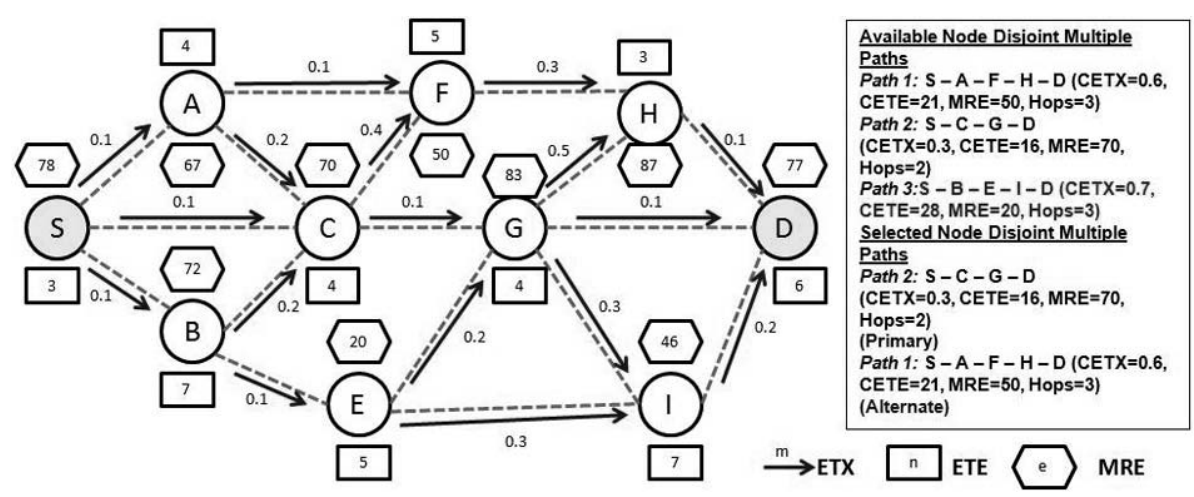

Figure 3. Route selection process of LR-EE-AOMDV protocol [16]. 
but less efficient than B-frames, as shown in Figure 4.

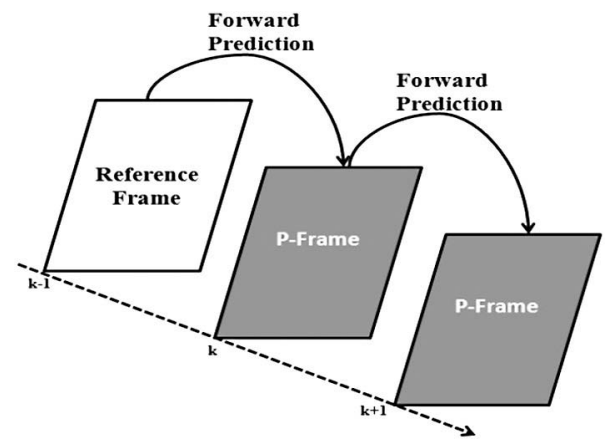

Figure 4. Concept of a P-Frame.

(iii) B-frames (Bi-directional) - frames in a video clip that are produced by the encoder using both forward and backward reference to previous/next I or P-frames for redundant picture information. B-frames are the most efficient frames of the three but they are not available in H.264's Baseline Profile, as shown in Figure 5.

Most broadcast qualitative applications have a propensity to use two consecutive $\mathrm{B}$ frames (I, $\mathrm{B}, \mathrm{B}, \mathrm{P}, \mathrm{B}, \mathrm{B}, \mathrm{P}, \ldots)$ for the ideal trade-off between compression efficiency and video quality. Figure 6 shows an example of MPEG video frame sequence. Quarter Common Intermedi-

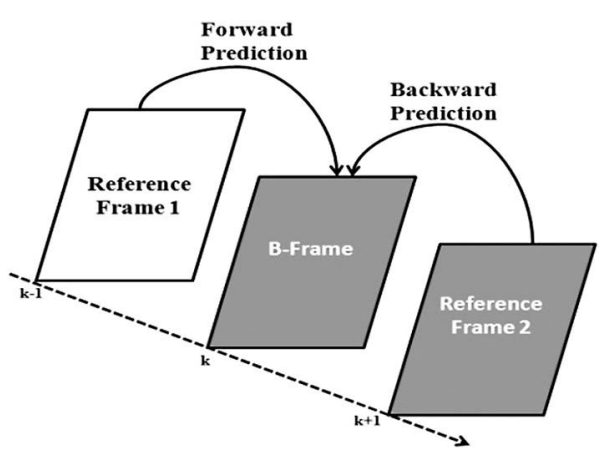

Figure 5. Concept of a B-Frame.

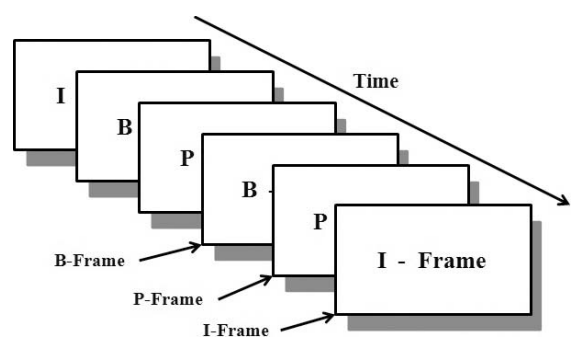

Figure 6. MPEG video frame types.

ate Format (QCIF) is selected because of its acceptable resolution efficiency and suitability in bit-rate and frame rate by providing $56 \mathrm{kbps}$ and 30 fps (frames per second) respectively.

The main advantage of $B$ frames is to enhance the coding efficiency. The $\mathrm{B}$ frames will result in less bits being coded overall in many cases

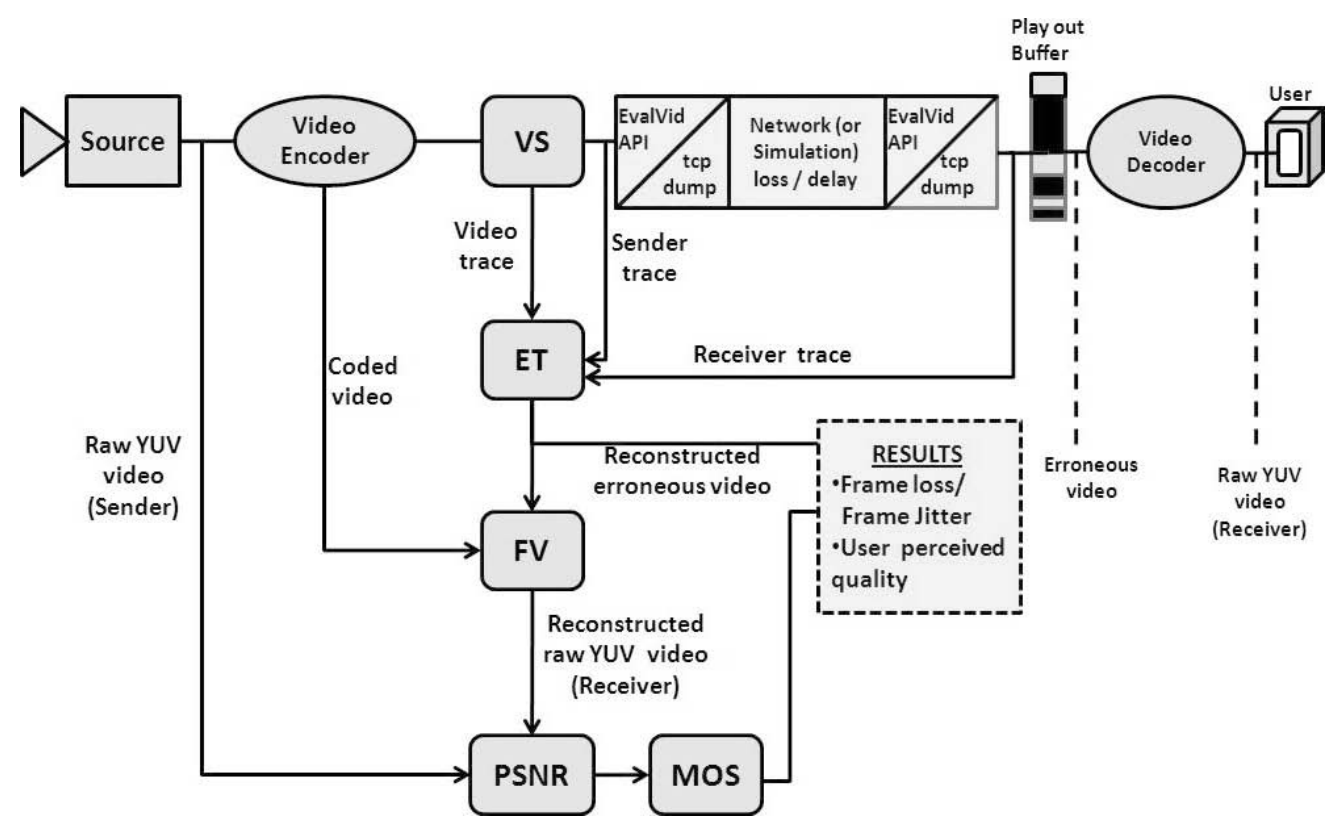

Figure 7. Structure of enhanced EvalVid framework [18]. 
and the quality can also be improved in the case of moving objects that reveal the hidden areas within a video sequence. Backward prediction allows the encoder to make more intelligent decisions on how to encode the video within these areas in a particular case. Since B frames are not used to predict future frames, the generated errors will not be propagated further within the sequence.

\subsection{Enhanced EvalVid Framework}

Enhanced EvalVid [18] is an extended framework and toolset of EvalVid [19], [20] for evaluating the quality of the H.264/MPEG-4 AVC video transmitted over a real or simulated communication network. This framework provides packet/frame loss rate, packet/frame jitter, PSNR, and MOS metrics for video quality assessment purposes. The structure of the enhanced EvalVid framework is shown in Figure 7. The main components of the enhanced EvalVid are described as follows:

Source - It can be a video, either in the YUV QCIF $(176 \times 144)$ or in the YUV CIF $(352 \times$ 288) formats.

Video Encoder and Video Decoder - The enhanced EvalVid supports three kinds of MPEG4 codecs such as NCTU codec [21], ffmpeg [22] and Xvid [23] but the original EvalVid supports only a single layer video coding. The focus of this investigation is NCTU codec for video coding purposes.

VS (Video Sender) - This component is used to read the compressed video file from the output of the video encoder. Each large video frame is fragmented into smaller segments, and then these segments are transmitted via UDP packets over a real or simulated network. This framework records the timestamp, the packet ID, and the packet payload size of each transmitted UDP packet in the sender trace file with the help of third-party tools such as tcp-dump [24] or win-dump [25] if the network is a real link.

ET (Evaluate Trace) - Once the video transmission is over, the information about the timestamp, the packet ID and the packet payload size available at the receiver have to be transported back to the sender in order to begin the evaluation task at the sender side. The ET component creates a frame/packet loss and frame/packet jitter report and generates a reconstructed video file based on the original encoded video file, the video trace file, the sender trace file and the receiver trace file which corresponds to the possibly corrupted video found at the receiver side as it would be reproduced to an end user.

FV (Fix Video) - Digital video quality assessment is performed frame by frame. Therefore, the total number of video frames at the receiver side, including the erroneous frames, must be the same as that of the original video at the sender side. If the codec cannot handle missing frames, the FV component is used to tackle this problem by inserting the last successfully decoded frame in the place of each lost frame as an error concealment technique [26].

\subsection{Integrated Structure of Enhanced EvalVid Framework with NS2}

Figure 8 illustrates the integrated structure of enhanced EvalVid with NS2. The enhanced EvalVid has the following three interface programs for communicating with NS2: MyTrafficTrace, My_UDP, and MyEvalvid_Sink:

(i) MyTrafficTrace agent extracts the frame type and the frame size of the video trace file generated from the traffic trace file, fragments the video frames into smaller segments, and sends these segments to the lower UDP layer at the appropriate time, according to the user settings specified in the simulation script file.

(ii) My_UDP is an extension of the UDP agent allowing the user to specify the output file that is the name of the sender trace file which records the timestamp of each transmitted packet, the packet ID, and the packet payload size. The task of the My UDP agent corresponds to the task that tools such as tcp-dump or win-dump perform in a real network environment.

(iii) MyEvalvid_Sink is the receiving agent for the fragmented video frame packets sent by My_UDP. This agent also records the timestamp, packet ID, and payload size of each received packet which have been kept in the user-specified receiver trace file.

As a result, the whole evaluation process starts from encoding the raw YUV video, and then the 


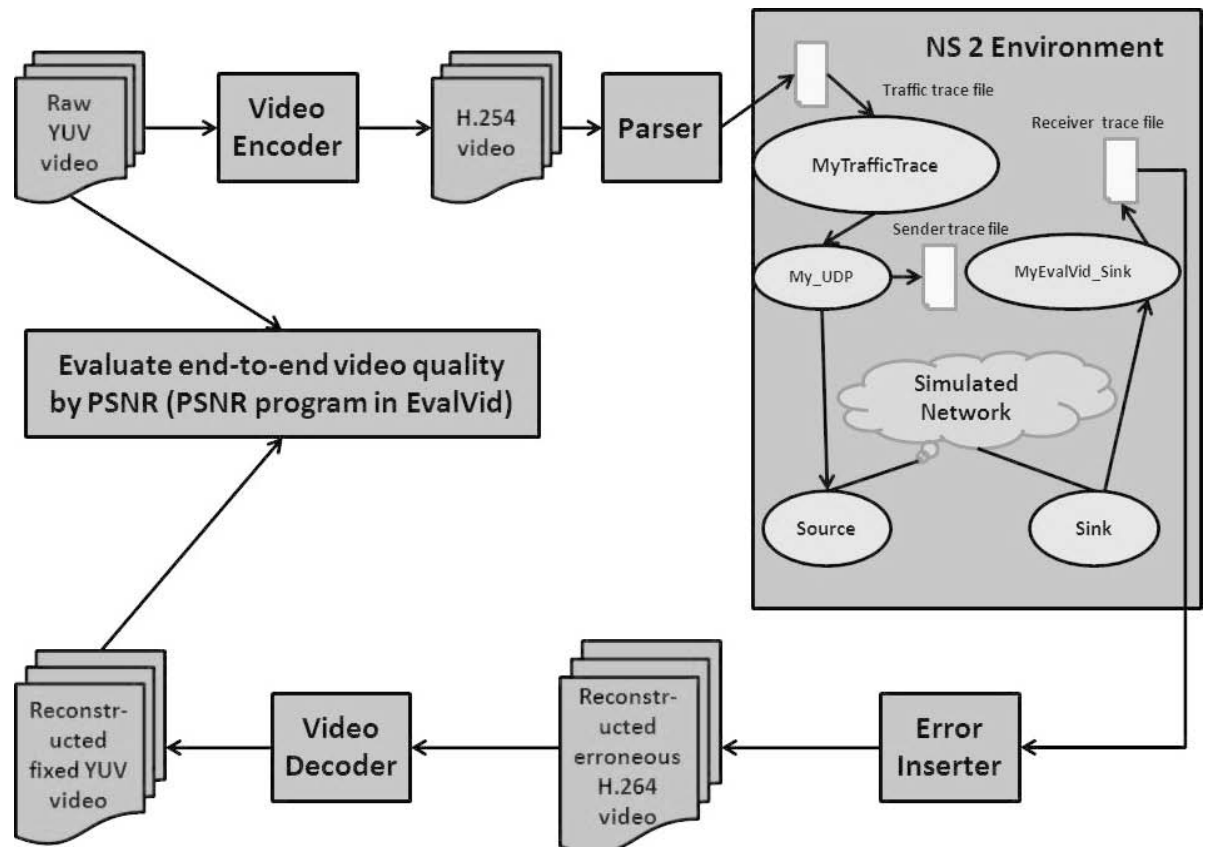

Figure 8. Integrated structure of the enhanced EvalVid with ns-2 [18].

VS program will read the compressed file and generate the traffic trace file. After simulation, the ET program produces the corrupted video file based on these three trace files and the original encoded video. Subsequently, the corrupted video is decoded and error concealed. Finally,

Table 4. Possible PSNR to MOS conversion under Absolute Category Rating (ACR) test.

\begin{tabular}{|c||c|}
\hline \multicolumn{1}{|c||}{ Parameter (s) } & Value (s) \\
\hline \hline Simulator & NS-2.34 \\
\hline MAC Types & 402.11 DCF \& 802.11 EDCF \\
\hline Simulation Area & $500 \mathrm{~m} \times 500 \mathrm{~m}$ \\
\hline Simulation Time & Wireless Channel \\
\hline Channel Type & OOMDV, OMMRE-AOMDV and LR-EE-AOMDV \\
\hline Routing Protocols & TwoRayGround \\
\hline Antenna Model & 2 Kbyte \\
\hline Radio Propagation Model & $1 \mathrm{Kbyte}$ \\
\hline Packet size & $100 \mathrm{Byte}$ \\
\hline Hello packet size & $176 \times 144(\mathrm{QCIF})$ \\
\hline Other control packet size & 0.2 \\
\hline Frame size & $56 \mathrm{kbps}$ \\
\hline Bits per pixel & $250 \mathrm{~ms}$ \\
\hline Bit rate & $500 \mathrm{~mW}$ \\
\hline Maximum tolerable delay & $50-60 \mathrm{~ms}$ \\
\hline Average codec power/packet &
\end{tabular}


the reconstructed fixed YUV video can be compared with the original raw YUV video to evaluate the end-to-end delivered video quality.

\section{Video Quality Metrics}

Quality of Service (QoS) and Quality of Experience $(\mathrm{QoS})$ are the qualitative measures of the videos which have to be delivered over wireless communication networks, but QoE reflects the user perception. The most widely used QoE metrics are as follows:

(i) Peak Signal-to-Noise Ratio (PSNR) - a traditional objective quality metric used to measure the video quality level based on the original and processed video sequences. PSNR of the frames with $\mathrm{M} \times \mathrm{N}$ pixels and 8 bits/sample is defined using Equation 1 as follows:

$P S N R=20 \log _{10}\left[\frac{255}{\sqrt{\frac{1}{M x N} \sum_{i=0}^{M-1} \sum_{j=0}^{N-1}\left\|Y_{S}(i, j)-Y d(i, j)\right\|^{2}}}\right]$

(ii) Mean Opinion Score (MOS) [27] - the most widely used subjective quality measure which measures the quality of a system by using "opinion score" in ITU-T Recommendation P.800. Depending on the quality factors to be assessed, MOS can be classified into assessments of listening quality and conversation quality. Possible PSNR to MOS conversion under Absolute Category Rating (ACR) [28] test is shown in Table 4. MOS can be assessed in terms of listening quality and conversation quality. The listening quality expresses the quality experienced by users when listening to a speech and the conversation quality expresses the quality experienced by users when taking part in a conversation.

\section{Experimental Results and Discussion}

We evaluate the performance of Link Reliable Energy Efficient Ad hoc On-demand Multipath Distance Vector (LR-EE-AOMDV) routing on H.264/MPEG-4 AVC video streaming un- der Distributed Coordination Function (DCF) or Distributed Channel Access (DCA) and Enhanced Distributed Coordination Function (EDCF) or Enhanced Distributed Channel Access (EDCA) using NS2.34 [29,30,31] and enhanced EvalVid framework [18]. Table 5 shows selected parameters for our simulation design.

Table 5. Possible PSNR to MOS conversion under Absolute Category Rating (ACR) test.

\begin{tabular}{|c||c|c|}
\hline PSNR [dB] & MOS & Category \\
\hline \hline$>37$ & 5 & Excellent \\
\hline $31-37$ & 4 & Good \\
\hline $25-31$ & 3 & Fair \\
\hline $20-25$ & 2 & Poor \\
\hline$<20$ & 1 & Bad \\
\hline
\end{tabular}

From Figure 9 and Figure 10, it is obvious that the Peak Signal to Noise Ratio (dB) of LR-EEAOMDV is better than the OMMRE-AOMDV and AOMDV routing protocols under both DCA and EDCA due to the selection of paths based on minimal hop count, CETX (CETX < 1 and CTEX $>0$ ) and CETE (CETE $<$ MRE). In AOMDV, the routes are selected based on only minimal hop count, which does not ensure link reliability and node efficiency. In OMMRE-AOMDV, the routes are selected based on only minimal hop count and maximized minimal residual energy, which does not ensure link reliability.

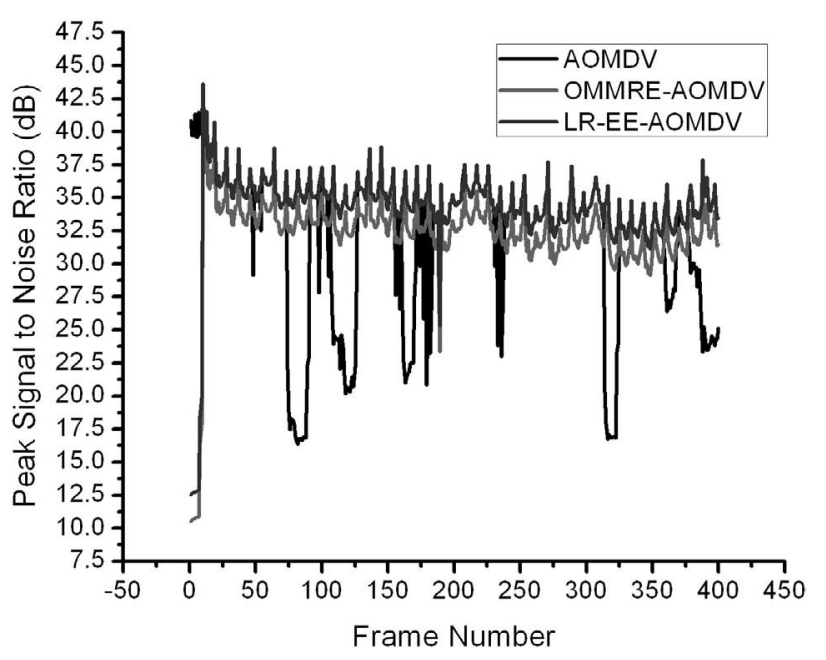

Figure 9. Peak signal to noise ratio (dB) under DCA. 


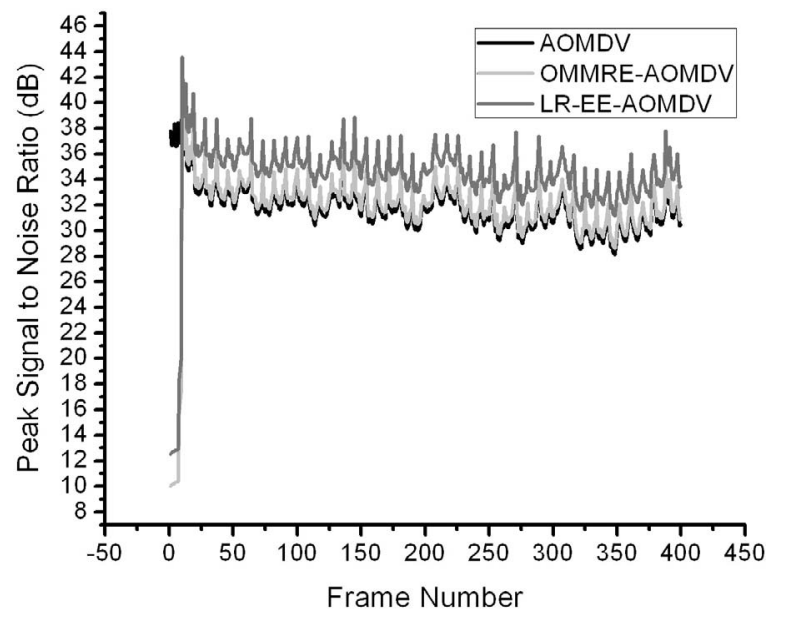

Figure 10. Peak signal to noise ratio (dB) under EDCA.

From Figure 11 and Figure 12, it is also seen that the Average PSNR and throughput of LR-EEAOMDV is better than the OMMRE-AOMDV and AOMDV routing protocols under both DCA and EDCA due to the selection of paths based on minimal hop count, CETX (CETX < 1 and CTEX $>0$ ) and CETE (CETE $<$ MRE). In AOMDV, the routes are selected based on only minimal hop count, which does not ensure link reliability and node efficiency. In OMMRE-AOMDV, the routes are selected based on only minimal hop count and maximized minimal residual energy, which does not ensure link reliability.

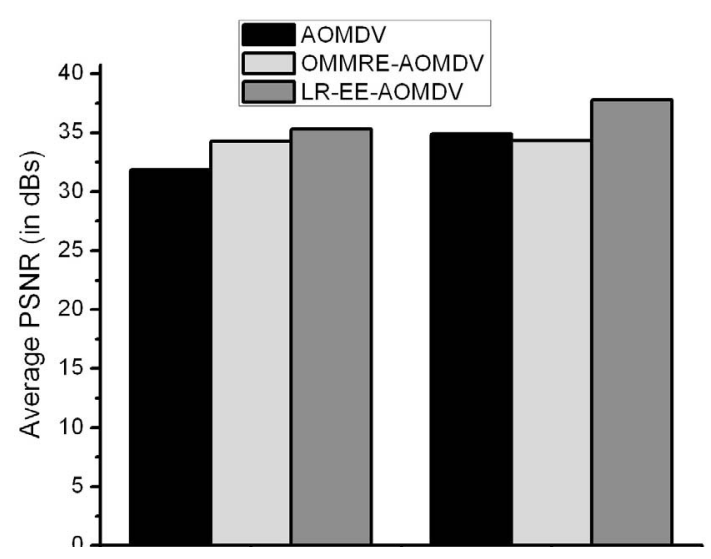

Figure 11. Average PSNR (in dBs).

From Figures 13, 14 and 15, it is also found that the end to end delay and average end to end delay of LR-EE-AOMDV are significantly re- duced in comparison with OMMRE-AOMDV and AOMDV routing protocols under both DCA and EDCA, due to the selection of paths based on minimal hop count, CETX (CETX < 1 and CTEX $>0$ ) and CETE (CETE $<$ MRE). In AOMDV, the routes are selected based on only minimal hop count, which does not ensure link reliability and node efficiency. In OMMRE-AOMDV, the routes are selected based on only minimal hop count and maximized minimal residual energy, which does not ensure link reliability.

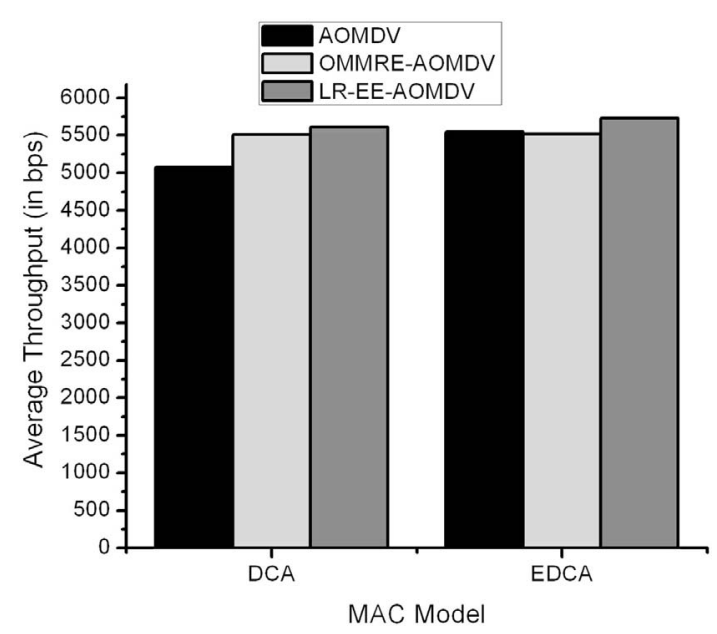

Figure 12. Average throughput (in bps).

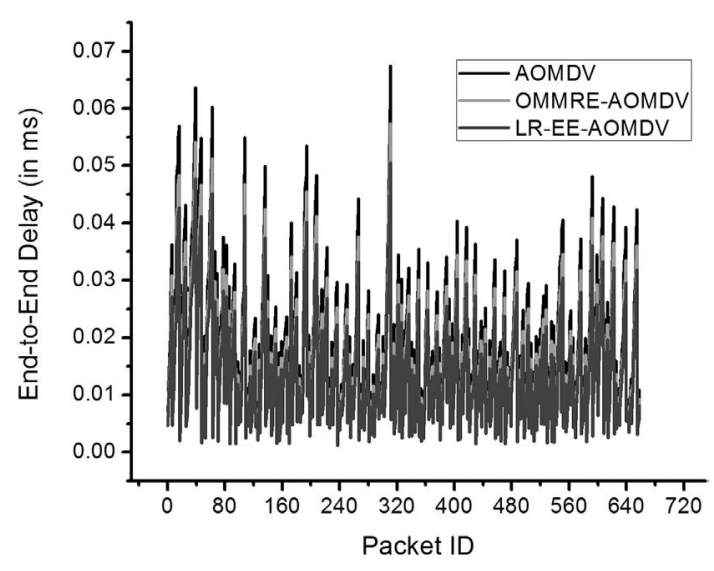

Figure 13. End to end delay (in ms) in DCA.

\section{Conclusions and Future Work}

Deploying real time applications like VoIP (Voice over IP) and videoconferencing in LongTerm Evolution (LTE) networks such as Inter- 


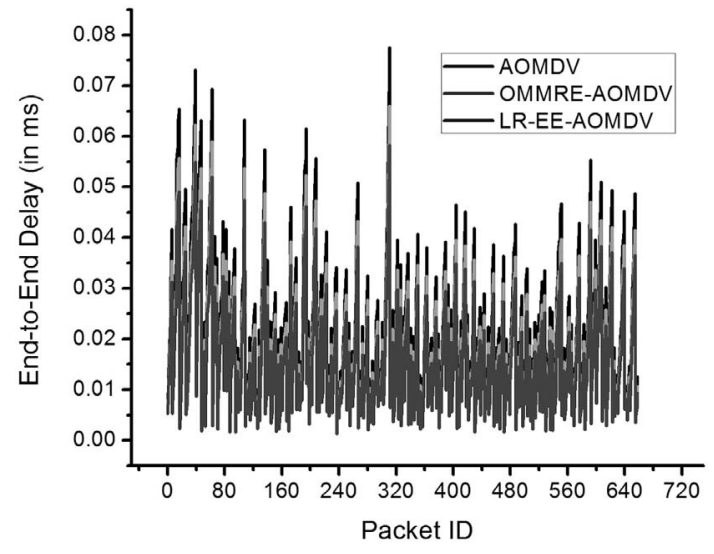

Figure 14. End to end delay (in ms) in EDCA.

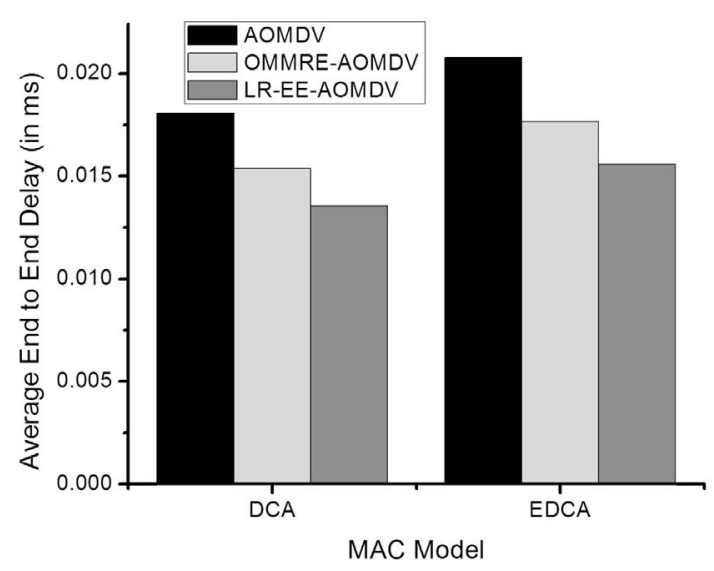

Figure 15. Average end to end delay (in ms).

net requires new challenges. Quality-of-Service (QoS) is a qualitative measure which can be used to ensure the video transmission quality in terms of packet delivery ratio, average endto-end delay, throughput, routing overhead, etc. Quality-of-Experience (QoE) is a qualitative measure of video frames transmitted over Internet subject to the experience of users. QoE includes Peak Signal-to-Noise Ratio (PSNR) and Mean Opinion Score (MOS).

In this paper, we evaluated the performance of Link Reliable Energy Efficient Ad hoc On-demand Multipath Distance Vector (LREE-AOMDV) routing on H.264/MPEG-4 Advanced Video Coding (AVC) video streaming under both Distributed Coordination Function (DCF) and Enhanced Distributed Coordination Function (EDCF) using NS2.34 and enhanced myEvalVid framework.
Since the LR-EE-AOMDV routing protocol selects the link-reliable energy-efficient multiple routes based on minimal hop count, CETX (CETX $<1$ and CTEX $>0$ ) and CETE (CETE < MRE), the Peak Signal to Noise Ratio (dB), Average PSNR and Throughput of LR-EE-AOMDV are better than the OMMRE-AOMDV and AOMDV routing protocols under both DCA and EDCA in Video streaming environment.

Since the LR-EE-AOMDV routing protocol selects the link-reliable energy-efficient multiple routes based on minimal hop count, CETX $($ CETX $<1$ and CTEX $>0)$ and CETE (CETE $<$ MRE), the end to end delay and average end to end delay of LR-EE-AOMDV are significantly reduced in comparison with OMMRE-AOMDV and AOMDV routing protocols under both DCA and EDCA in Video streaming environment.

In future, the LR-EE-AOMDV will be enhanced to adopt with WiMAX and will also be evaluated considering Scalable Video Coding (SVC) strategies under cognitive radio or delay/disruption tolerant network environments.

\section{References}

[1] E. M. Royer and C.-K. Toh, "A Review of Current Routing Protocols for Ad-Hoc Mobile Wireless Networks", IEEE Personal Communications, vol. 6, no. 2, pp. 46-55, 1999.

http://ieeexplore.ieee.org/document/760423 http://dx.doi.org/10.1109/98.760423

[2] M. Abolhasan et al., "A review of routing protocols for mobile ad hoc networks", Ad Hoc Networks, vol. 2, no. 1, pp. 1-22, 2004. http://dx.doi.org/10.1016/S1570-8705(03)00043-X

[3] 3GPP TS 136.300, LTE; Evolved Universal Terrestrial Radio Access (E-UTRA) and Evolved Universal Terrestrial Radio Access Network (E-UTRAN); Overall description; Stage 2 (Release 10), version 10.7.0, Mar. 2012.

[4] H. Chen et al., "End-to-end quality adaptation scheme Based on QoE prediction for video streaming service in LTE networks", in 11th International Symposium on Modeling \& Optimization in Mobile, Ad Hoc \& Wireless Networks (WiOpt), pp. 627-633, 2013.

http://ieeexplore.ieee.org/abstract/document/6576411/

[5] ITU - International Telecommunication Union, "Definition of Quality of Experience", Ref: TD 109rev2 (PLEN/12), Jan. 2007. 
[6] ITU - T Rec. H.264 and ISO/IEC 14496-10 (MPEG4-AVC), "Advanced Video Coding for Generic Audiovisual Services", v1, May, 2003; v2, Jan. 2004; v3 (with FRExt), Sept. 2004; v4, July 2005 .

[7] M. K. Marina and S. R. Das, "Ad hoc on-demand multipath distance vector routing, International Journal of Wireless Communications And Mobile Computing", vol. 6, no. 7, pp. 969-988, 2006. http://dx.doi.org/10.1002/wcm.432

[8] C. E. Perkins and E. M. Royer, "Ad hoc on-demand distance vector routing", Proc. of the IEEE Workshop on Mobile Computing Systems and Applications, New Orleans, LA, pp. 90-100, 1999. http://dx.doi.org/10.17487/RFC3561

[9] S. Tang et al., "A link heterogeneity-aware on-demand routing (LHAOR) protocol utilizing local update and RSSI information", IEICE TRANSACTIONS on Communications, vol. E88-B, no.9, pp. 3588-3597, 2005.

http://dx.doi.org/10.1093/ietcom/e88-b.9.3588

[10] S. Khimsara et al., "AM-AOMDV: Adaptive Multi-metric Ad-hoc On Demand Multipath Distance Vector Routing, Workshop on Cross-layer Design in Wireless Mobile Ad hoc Networks", First International Conference on Ad Hoc Networks (AdHocNets'09), Niagara Falls, Canada, pp. 884-895, 2009.

http://link.springer.com/chapter/10.1007/ 978-3-642-11723-7 61

http://dx.doi.org/10.1007/978-3-642-11723-7_61

[11] P. Periyasamy and E. Karthikeyan, "Energy Optimized Ad hoc on-Demand Multipath Routing Protocol for Mobile Ad hoc Networks", International Journal of Intelligent Systems and Applications (IJISA), vol.6, no.11, pp. 36-41, 2014. http://dx.doi.org/10.5815/ijisa.2014.11.05

[12] Y. Liu et al., "Energy efficient on demand multipath routing protocol for multi-hop ad hoc networks", 10th IEEE International Symposium on Spread Spectrum and Applications (ISSSTA-08), Italy, pp. 592-597, 2008.

http://ieeexplore.ieee.org/abstract/document/ 4621471/

http://dx.doi.org/10.1109/ISSSTA.2008.112

[13] D. S. J. D Couto et al., "A high-throughput path metric for multi-hop wireless routing", Wireless Networks, vol. 11, no. 4, pp. 419-434, 2005. http://dx.doi.org/10.1007/s11276-005-1766-z

[14] M.-K. Kim and H. P. Ngo, "Multipath-Based Reliable Routing Protocol with Fast-Recovery of Failures on MANETs", Future Information Technology, Application and Service, Lecture Notes in Electrical Engineering, Springer, pp. 81-90, 2012.

http://link.springer.com/chapter/10.1007/ 978-94-007-4516-2 9

http://dx.doi.org/10.1007/978-94-007-4516-2_9
[15] Y. L. Jin et al., "Expected Transmission Energy Route Metric for Wireless Mesh Senor Networks", International Journal of Digital Multimedia Broadcasting, Hindawi Publishing Corporation, pp. 1-7, 2011.

http://dx.doi.org/10.1155/2011/947396

[16] P. Periyasamy and E. Karthikeyan, "Link reliable energy-efficient AOMDV routing protocol for mobile ad hoc networks", International Journal of Ad Hoc and Ubiquitous Computing (IJAHUC), Inderscience, 2015. (Article in Press). http://dx.doi.org/10.1504/IJAHUC.2015.10001745

[17] J. Ostermann et al., "Video coding with H.264/ AVC: tools, performance, and complexity", Circuits and Systems Magazine, IEEE, vol. 4, no. 1, pp. 7-28, 2004.

http://ieeexplore.ieee.org/abstract/document/ 1286980/

http://dx.doi.org/10.1109/MCAS.2004.1286980

[18] C. H. Ke et al., "An Evaluation Framework for More Realistic Simulations of MPEG Video Transmission", Journal of Information Science and Engineering, vol. 24, no. 2, pp. 425-440, 2008.

http://www.iis.sinica.edu.tw/page/jise/2008/ 200803_07.pdf

[19] J. Klaue et al., "EvalVid - A framework for video transmission and quality evaluation", Proc. of the International Conference on Modelling Techniques and Tools for Computer Performance Evaluation, pp. 255-272, 2003.

http://link.springer.com/chapter/10.1007/ 978-3-540-45232-4 16 http://dx.doi.org/10.1007/978-3-540-45232-4_16

[20] Enhanced EvalVid Framework: http://hpds.ee.ncku.edu.tw/ smallko/ns2/ Evalvid_in_NS2.htm.

[21] NCTU codec, http://megaera.ee.nctu.edu.tw/mpeg.

[22] ffmpeg, http://ffmpeg.sourceforge.net/index.php

[23] Xvid, http://www.Xvid.org/

[24] tcp-dump, http://www.tcpdump.org

[25] win-dump, http://windump.polito.it

[26] Y. Wang and Q. F. Zhu, "Error control and concealment for video communication: a review", in Proceedings of the IEEE, vol. 86, pp. 974-997, 1998.

[27] Speech quality assessment methods, http://www.ntt.co.jp/qos/qoe/eng/technology/ sound/index.html 
[28] Video quality assessment methods, http://www.ntt.co.jp/qos/qoe/eng/technology/ visual/index.html

[29] The Network Simulator ns-allinone-2.34, http://www.isi.edu/nsnam/ns/

[30] Kevin Fall and K. Varadhan, "The ns Manual", University of Southern California, Information Sciences Institute (ISI), http://www.isi.edu/nsnam/ns/ns-documentation. html.

[31] NS-2 with Wireless and Mobility Extensions, http://monarch.cs.rice.edu/cmu-ns.html
Contact addresses:

Periyasamy Pitchaipillai Department of Computer Science and Applications Sree Saraswathi Thyagaraja College Pollachi, 642107

Tamil Nadu, India e-mail: pereee@yahoo.com

Karthikeyan Eswaramoorthy Department of Computer Science Government Arts College

Udumalpet, 642126

Tamil Nadu, India

e-mail: e_karthi@yahoo.com
Received: May 2016

Revised: December 2016 Accepted: January 2017
Periyasamy Pitchaiplllai has obtained his $\mathrm{PhD}$ in Computer Science from Government Arts College, Udumalpet, Tamil Nadu, India (affiliated to Bharathiar University, Coimbatore) in December 2016 and is presently working as Assistant Professor of Computer Science and Applications at Sree Saraswathi Thyagaraja College, Pollachi, Tamil Nadu, India. His research interests include routing protocol design and quality of service (QoS) enhancement of wireless networks such as MANETs, VANETs, CRAHNs and DTNs. He has published 12 papers in international journals and conference proceedings. He has also attended more than 10 conferences at national and international levels. $\mathrm{He}$ is a reviewer in Springer - Wireless Personal Communications Journal, KSII Transactions on Internet and Information Systems and Journal of Communications and Information Networks.

KARTHIKEYAN EswaRAmoorthy obtained his PhD in Computer Science from Gandhigram University, Dindigul, Tamil Nadu, India in 2008 and is presently working as Assistant Professor of Computer Science at Government Arts College, Udumalpet, Tamil Nadu, India. His research interests include network security and cryptography, MANET routing, congestion control, and advanced networking. He has published $27 \mathrm{pa}-$ pers in international journals and attended more than 15 conferences at national and international levels. He has also published a book entitled Text Book on C: Fundamentals, Data Structures and Programming by PHI. He is a life member of CSI, CRSI, IASCT and Editor-in-Chief of IJANA. 